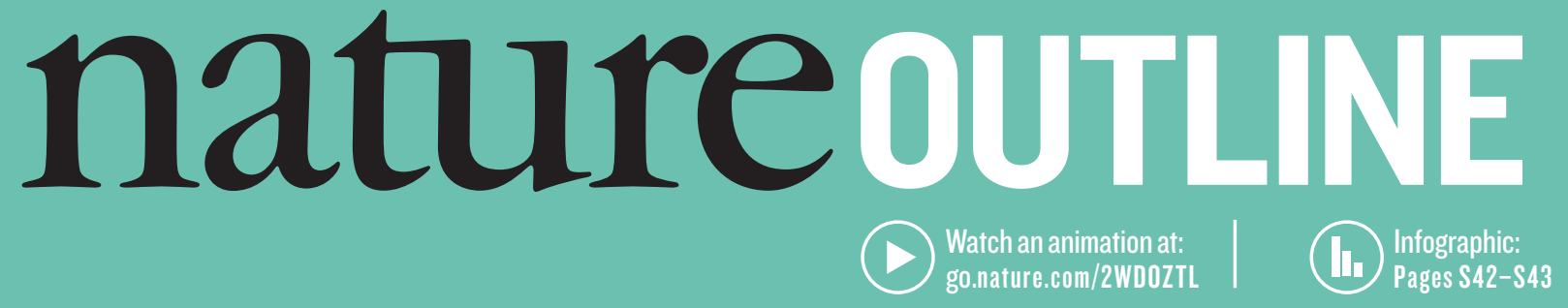

\title{
CRITICAL LIMB ISCHAEMIA: ARTERY REPAIR
}

\section{Can regenerative medicine help to avoid leg amputation in patients with critical limb ischaemia?}

\section{BY DAVID HOLMES}

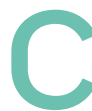

ritical limb ischaemia (CLI) is a cardiovascular condition at the most severe end of the spectrum of peripheral arterial disease. In CLI, blockages within the arteries restrict the flow of blood to the affected limbs - typically the lower extremities. Severe pain in the feet or toes ensues, and further complications include ulcers and gangrenous wounds on the legs and feet. The negative impact of CLI on a patient's quality of life is profound, but of greater concern is the associated high risk of amputation of the affected limb, or even death.

More than half of people with CLI die within 5 years of their diagnosis (M. Teraa et al. J. Am. Heart Assoc. 5, e002938; 2016), usually from a heart attack or stroke. The estimated annual incidence of CLI in Europe and the United States is 500-1,000 cases per 1 million people (L. Norgren et al. J. Vasc. Surg. 45, S5-S67; 2007). As populations age and the prevalence of risk factors such as obesity and diabetes continues to grow across the world, so too will the burden of CLI.

The projected increase in the prevalence of CLI makes the need to address a shortfall in treatment options all the more pressing. At present, treatments are limited to surgical interventions that aim to bypass or remove the arterial blockage. Such interventions have good rates of success in patients who are eligible to receive them. But, unfortunately, there is a sizeable minority - up to $30 \%$ of people with CLI - for whom these treatments are not possible, either because the affected blood vessels are too badly calcified, or the patient has another condition that prevents them. The only current alternative is limb amputation.

There is, however, a glimmer of hope on the horizon. Regenerative therapies have the potential to induce the growth of new blood vessels - angiogenesis - and therefore provide hope of restoring blood supply to the affected limbs. In this Outline, we look at some of the experimental regenerative therapies under development, one of the most promising of which involves cells that express the protein CD34.

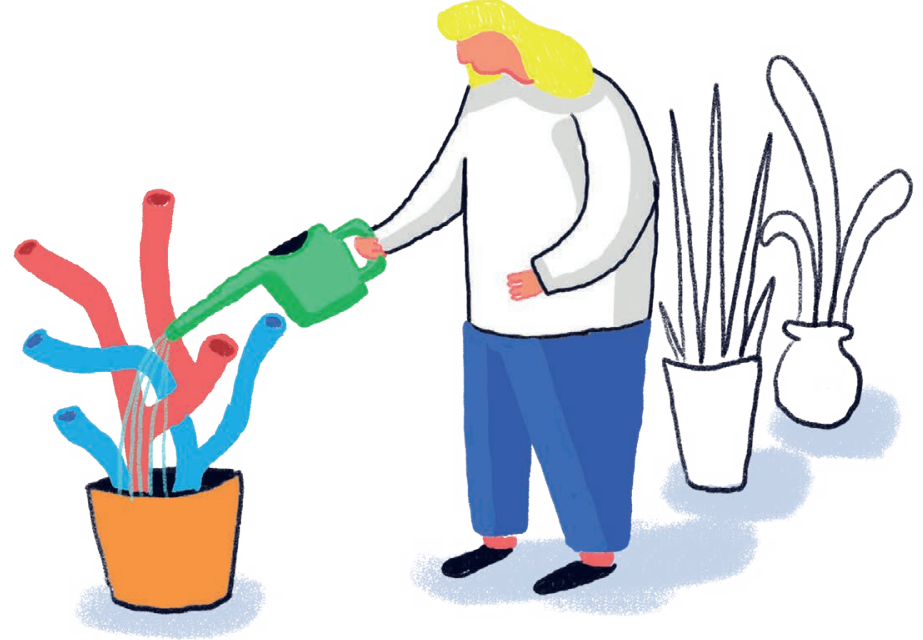

CD34 is found on the surface of endothelial progenitor cells, which are capable of migrating to sites of restricted blood flow and promoting angiogenesis. Although the function of CD34 is not known, it is possible to use antibodies to isolate cells that express the protein from samples of blood taken from people with CLI. A purified suspension of these cells is then injected into the affected limb. Early-phase clinical trials have shown that the treatment is safe and leads to improvements in wound healing. However, a much larger study will be required to determine whether this CD34 cell therapy can provide a considerable improvement in the length of time that people with CLI can go without needing to have a limb amputated. A research group in Japan has plans to start such a trial later this year. Meanwhile, preclinical studies in animal models of CLI have shown the potential benefits of combining CD34 cell therapy with growth factors that promote angiogenesis, together with new techniques for priming cells that express CD34 to increase their survival in ischaemic tissue. There is yet hope that, for many people, a diagnosis of CLI need not mean a loss of limb.

Nature is pleased to acknowledge the financial support of the Translational Research Informatics Center (TRI). As always, Nature retains sole responsibility for all editorial content.

David Holmes is a science writer in the United Kingdom.

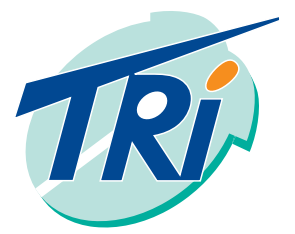

